\title{
Kernos
}

Revue internationale et pluridisciplinaire de religion grecque antique

$33 \mid 2020$

Varia

\section{Tracking Hermes, Pursuing Mercury}

\section{Dominique Jaillard}

\section{CpenEdition \\ Journals}

\section{Édition électronique}

URL : https://journals.openedition.org/kernos/3486

DOI : 10.4000/kernos.3486

ISSN : 2034-7871

\section{Éditeur}

Centre international d'étude de la religion grecque antique

\section{Édition imprimée}

Date de publication : 31 décembre 2020

Pagination : 316-318

ISBN : 978-2-87562-264-8

ISSN : 0776-3824

\section{Référence électronique}

Dominique Jaillard, «Tracking Hermes, Pursuing Mercury », Kernos [En ligne], 33 | 2020, mis en ligne le 31 décembre 2020, consulté le 02 décembre 2022. URL : http://journals.openedition.org/kernos/3486 ; DOI : https://doi.org/10.4000/kernos.3486

Ce document a été généré automatiquement le 2 décembre 2022

Tous droits réservés 


\title{
Tracking Hermes, Pursuing Mercury
}

\author{
Dominique Jaillard
}

\section{RÉFÉRENCE}

John F. MILLER, Jenny STRAuSS CLAY (dir.), Tracking Hermes, Pursuing Mercury, Oxford, Oxford University Press, 2019. 1 vol. $16 \times 24$ cm, 378 p. ISBN : 978-0-19-877734-2.

1 Un colloque de l'Université de Virginie (2014) est à l'origine de ce collectif qui se propose d'explorer, au fil de ses vingt contributions, les multiples facettes d'Hermès et de Mercure selon une approche interdisciplinaire, seule en mesure d'appréhender dans toute sa complexité une figure que les éditeurs décrivent comme la «la plus insaisissable, versatile et ambiguë des divinités antiques»(p.1). Sont mis à contribution «littératures" grecque et latine (épopée, poésie lyrique, théâtre), épigraphie, culte et religion, iconographie, sculpture. Partant du constat que les figures d'Hermès / Mercure sont, en dépit d'un ensemble d'études récentes, largement « sousétudiées ", l'introduction pointe un manque : «up to now there has been no attempt to discuss in a coherent manner the surprising variety of his literary, cultic, and artistic manifestations » (p. 2). Par son interdisciplinarité, le volume se veut un modèle pour de futures investigations (p. 2).

2 Les résultats de cette enquête multidimensionnelle sont regroupés en neuf chapitres « pinpointing some important aspects». La première partie (Son, Father, Brother) considère Hermès dans ses relations familiales, en écho à l'Hymne homérique dont le récit est centré sur la reconnaissance de ses prérogatives que le petit dieu obtient, de haute lutte, de son père et son frère Apollon. Les contributions d'A. Shapiro et de C. Laferrière étudient respectivement les relations d'Hermès avec sa mère Maia (dans leurs variations), et avec Pan et les nymphes, en prenant prioritairement en compte un corpus de représentations figurées (respectivement les vases d'époque archaïque et des reliefs votifs $\mathrm{du} \mathrm{IV} \mathrm{e}^{\mathrm{e}} \mathrm{s}$.). J. Larson examine les similarités entre le vol par Hermès du troupeau d'Apollon et celui de son trépied par Héraclès. 
3 La deuxième partie considère Hermès en tant que «trickster ». J. Strauss Clay analyse les rôles d'Hermès dans les poèmes homériques et les homologies qui peuvent être établies tant entre ces «doubles" divins et humains que sont les "polytropes " Hermès et Ulysse qu'entre les puissances de séduction qui traversent à la fois la parole du dieu et celle du poète épique. La contribution d'A. Campra et de C. Nobili interroge les rapports entre Hermès et la poésie iambique et lit l'Hymne homérique comme «la préhistoire mythique du genre iambique» (p. 79). La partie III (Comic) recense et interroge les rôles d'Hermès dans la comédie grecque (S. Beta) et romaine (E. Moodle), le dieu pouvant en quelque manière être considéré " as an embodiment of the comic genre » (p. 5 et 117).

4 La partie IV (Erotic) est également construite comme une mise en perspective entre Grèce et Rome. J. Farrell marque l'écart entre la figure ithyphallique du dieu et la relative sagesse de sa « vie amoureuse » dans les représentations littéraires, soulignant son rôle d'intermédiaire bienveillant entre les sexes et pointant les "double sens" sexuellement marqués avec lesquels joue l'Hymne homérique. M. Myers examine les rapports d'intertextualité entre Horace et Alcée et entre Ovide et l'Hymne homérique et interroge les superpositions et relations entre Mercure et Cupidon.

5 Les parties V et VI se concentrent sur Mercure, la V dans son rôle de médiateur, la VI dans ses rapports au commerce et aux échanges, tout en interrogeant ses relations avec des sous-textes grecs. La complexité de ces derniers ressort tout particulièrement de l'étude du Mercure d'Horace que propose S.-J. Harrison. La construction des liens privilégiés du dieu avec le poète et avec Octave suppose une sélection subtile et assumée de ses traits les plus pertinents dont la dimension politique ressort fortement en contexte augustéen. S. Casali, « Crossing the Borders. Vergil' Intertextual Mercury ", explore les relations de Mercure à la tromperie et à la vérité au chant IV de l'Énéide: les mensonges $\mathrm{du}$ dieu fonctionnent comme révélateur du caractère éminemment dangereux de la reine carthaginoise. Le lien marqué du Mercure romain avec le commerce (un aspect des échanges relevant des champs de compétence de l'Hermès grec) est étudié par D. Mac Rae, « Mercury and Materialism ", à partir des images des tabernae pompéiennes. Th. Biggs considère les dimensions maritimes de Mercure dans leur possible lien avec «the positive valuation of Rome's sea power in the wake of its victory over Carthage, before elite attitudes modulated toward a denunciation of maritime trade ».

6 La partie VII (Greek Religion and Cult) comporte trois contributions. Celle d'H. Collard, "Communicating with the Divine: Herms in Attic Vase painting", rouvre la question «du rôle, de la signification et de la fonction des piliers hermaïques» (p. 227). La profusion des images de l'hermès sur les vases attiques, notamment dans le contexte de scènes rituelles et sacrificielles, reflète moins la diffusion des cultes d'Hermès que la capacité du pilier hermaïque - «perçu et utilisé comme instrument rituel» - «à rendre la présence divine manifeste et à transformer l'espace dans lequel il apparaît en un lieu favorable à l'établissement d'un contact avec les dieux» (p. 242), et «de la relation qui convient» (p. 241). De l'examen des dédicaces à Hermès entre époque archaïque et époque hellénistique que propose J. Wallensten («Hermes as visible in Votive Inscriptions»), ressort "a polyvalent Hermes of many varied local and contextual characteristics, which coexists with the Hermes of Panhellenic Myth» (en lien notamment avec les échanges, les voyageurs, les magistrats, le gymnase). La variété des dédicataires et des puissances divines associées rend d'autant plus intrigante l'absence des femmes. L'A. souligne qu'aux époques hellénistique tardive et romaine, l'appellatif divin Neos Hermes est le seul à être appliqué à des humains qui ne soient ni des rois ni 
des membres de la famille impériale, en lien peut-être avec le « caractère du dieu » et l'aisance avec laquelle il franchit les limites du mortel et de l'immortel (p.254). En examinant dans son «Hermes, Kyllene, Samothrace and the Sea ", "a combination of image and function of which he (Hermès) was a part, rather than only a name, capable of many permutations ", S. Blakely enrichit la complexité des figures de l'Hermès de Samothrace dans leur relation à la mer et à la protection des navigateurs en pointant la place qu'y tient l'Hermès du Cyllène et son type iconographique.

7 La partie VIII (Egypt) étudie des hymnes associés à Hermès dans des papyri grecs d'Égypte. L.M. Bortolani lie ceux des Papyri magiques grecs à l'activité de spécialistes rituels "with an Egyptian cultural background» (p. 303) lors d'une phase relativement ancienne de l'interpretatio graeca de Thot. L'analyse de «l'éloge de la figue» (P.Oxy. 17. 2084) proposée par A. Vergatos éclaire son contexte performatif, les divers aspects d'Hermès qui y sont incorporés et la complexité des sous-textes impliqués.

8 La dernière partie (IX : Cosmic) s'ouvre sur une courte mais importante contribution de N. Reggiani : "Rethinking Hermes. Cosmic Justice and Proportional Distributions », dans laquelle les liens du dieu avec Moira, sa position dans les processus d'attribution et de distribution des parts, et sa capacité à les reconfigurer selon une proportion juste, sont mis en rapport avec les articulations complexes qui se laissent repérer entre les différentes modalités et sphères d'action du dieu, notamment ses relations à un espace discontinu qualitativement différencié, à la parole et au silence, au lancer des cailloux et des sorts... Ce qui ouvre aussi sur deux questions : en quoi Hermès est-il un dieu « pas comme les autres? (p. 326); comment penser autrement du «cosmique», je dirais, pour ma part, en clef hermaïque ? La contribution de H. Versnel, « Great Hermes. Three Ways toward Stardom ", s'emploie à rendre raison des transformations du "little, very popular, but relatively low ranking deity into an eminent god, glorified in matchless superlative terms» (p.338). L'ascension d'Hermès est expliquée en regard de "phénomènes analogues dans des tendances religieuses et culturelles contemporaines » (p. 338) et à partir des contextes et occasions qui, à défaut de faire le larron, ont fait le chef ${ }^{1}$.

L'ensemble des choix méthodologiques de cette dernière contribution exigerait une discussion longue et serrée qui n'a pas sa place ici. Sa position en conclusion du volume tend néanmoins à conférer à ce dernier une certaine coloration. Comment appréhender l'histoire d'un dieu? Appréhender une diversité et des transformations dont le sens obéit à des logiques autres, suppose d'autres agencements? Selon quels critères, par exemple, qualifions-nous un dieu de « mineur » ? S'il est un privilège d'Hermès - pour l'historien des religions antiques cette fois - c'est d'être un réactif de premier choix à l'inadéquation et aux limites de nos catégories. En quoi son action exige-t-elle cette relative invisibilité, cette tenue à distance de la boulē de Zeus qu'explore l'Hymne homérique? Et à rebours, en quel sens exact en vient-il à être dit pantokratōr ${ }^{2}$ ? $\mathrm{H}$. Versnel pointe dans l'épigramme crétoise le contexte du sanctuaire, l'enjeu rituel local, des pratiques nouvelles de l'époque impériale. Dont acte. Mais va-t-il de soi ( $w e$ have no doubt », p. 342), en lien avec l'usage de cet « extraordinary predicate » (p. 340), que " the personal, pious and submissive religiosity of the adorant Salvius Menas » est « the key to the god' 'stairway to heaven'» (p. 342) ? Et est-ce bien de cela qu'il s'agit?

Par delà l'incontestable richesse des contributions singulières dans le détail duquel nous aimerions qu'il nous fût loisible d'entrer, l'intérêt premier du volume en tant que livre tient à son effort de mise en perspective des multiples dimensions du dieu « against their background as adumbrated in the (Homeric) Hymn» (p. 2), à la confrontation 
d'approches variées relevant de diverses disciplines. Il réussit ainsi à mettre en évidence des « signposts for further pursuits » (p.10) que se doit de ne pas négliger toute tentative de cartographie des positions du dieu dans la dynamique de leurs agencements. Il suggère ces "zig-zag path, continuities that cross generic and temporal boundaries, detours and byways»(p.10) qui interdisent de tracer lignes simples ou frontières étanches entre le «naughty babe » de l'Hymne homérique et l'Égypte hellénisée (p. 10). On peut en revanche douter qu'il ait rempli son objectif de discuter «de manière cohérente la surprenante variété des manifestations » du dieu (p. 2), tant les approches réunies relèvent de questionnements, de problématiques, de méthodologies dont la nature ou les divergences n'autorisent pas à aller au-delà de l'inventaire de la multiplicité et de mises en perspective suggestives. Un travail réflexif et critique sur un ensemble de catégories et de concepts donnés comme allant de soi, ne citons que l'idée d'un "god's character", de son "evolution ", d'un "Mercure alter ego d'Hermès", pourrait constituer un des prolongements souhaités par les éditeurs. Une discussion s'imposerait quant aux articulations entre ce qui relève de savoirs partagés et de la "diffusion» ou de la circulation de motifs. Et du point de vue de l'anthropologue historien des polythéismes antiques qu'est l'auteur de la présente recension, le repérage des multiples positions du dieu appellerait leur insertion - problématisée à partir des pratiques qui les mobilisent - dans le tissu polythéiste et dans la dynamique de ses reconfigurations.

\section{NOTES}

1. "And in the case of Hermes it was the occasion that made the chief » (p. 352).

2. ICret. II 27. 2 (Versnel, p. 339).

\section{AUTEURS}

DOMINIQUE JAILLARD

Université de Genève 